\title{
Cross-polarization and sidelobe suppression in dual linear polarization antenna arrays
}

\author{
Woelders, Kim; Granholm, Johan
}

Published in:

I E E E Transactions on Antennas and Propagation

Link to article, DOI:

10.1109/8.650190

Publication date:

1997

Document Version

Publisher's PDF, also known as Version of record

Link back to DTU Orbit

Citation (APA):

Woelders, K., \& Granholm, J. (1997). Cross-polarization and sidelobe suppression in dual linear polarization antenna arrays. I E E E Transactions on Antennas and Propagation, 45(12), 1727 - 1740.

https://doi.org/10.1109/8.650190

\section{General rights}

Copyright and moral rights for the publications made accessible in the public portal are retained by the authors and/or other copyright owners and it is a condition of accessing publications that users recognise and abide by the legal requirements associated with these rights.

- Users may download and print one copy of any publication from the public portal for the purpose of private study or research.

- You may not further distribute the material or use it for any profit-making activity or commercial gain

- You may freely distribute the URL identifying the publication in the public portal

If you believe that this document breaches copyright please contact us providing details, and we will remove access to the work immediately and investigate your claim. 


\title{
Cross-Polarization and Sidelobe Suppression in Dual Linear Polarization Antenna Arrays
}

\author{
Kim Woelders and Johan Granholm, Member, IEEE
}

\begin{abstract}
In recent years, there has been an increasing interest in dual linear polarization antennas for various purposes, e.g. polarimetric synthetic aperture radar (SAR) imaging. A key design goal for dual polarization antennas is to obtain a high cross-polarization suppression. When using standard techniques for improving the cross-polarization suppression in dual linear polarization antenna arrays undesired sidelobes appear. This paper describes the properties of some known cross-polarization suppression methods and presents a new method for obtaining high cross-polarization suppression and simultaneously avoiding undesired sidelobes.
\end{abstract}

Index Terms-Antenna arrays, dual polarization antennas.

\section{INTRODUCTION}

A NY dual polarization antenna is fed using two "ports." We will consider antennas for which the desired function is that the radiation pattern is horizontally polarized when applying a signal to one port and vertically polarized when applying a signal to the other port. The exact definition of horizontal and vertical polarization is not essential in this context. Definition 3 of [1] has been adopted in the present work.

In an antenna array, a feeding network distributes the signal from the antenna ports to the individual antenna element ports. The signal applied to the individual antenna element is designated the element excitation. The radiation pattern of the antenna array is determined from the excitations, the spatial locations, and the radiation patterns of the individual antenna elements (neglecting mutual coupling effects).

The elements of the antenna array can be of any type that can be excited on two orthogonal polarizations independently. In all "real" antenna elements the fields generated by exciting each of the feed ports will not be purely orthogonal due to a finite amount of cross polarization. The discussions below are illustrated using dual linearly polarized square microstrip patch elements with one feed probe per polarization (see Fig. 1). The dots mark the locations of the feed probes (ports). A signal applied at an $H$-port will primarily generate a horizontally polarized field and a signal applied at a $V$-port will primarily generate a vertically polarized field.

We will consider antenna arrays consisting of a number of identical $2 \times 2$ element subarrays with different configurations. The different $2 \times 2$ element subarray configurations are shown in Fig. 1. For each configuration it is shown how a particular

Manuscript received February 15, 1997; revised June 16, 1997. This work was supported by the Danish National Research Foundation.

The authors are with the Department of Electromagnetic Systems (formerly, the Electromagnetics Institute), Danish Center for Remote Sensing, Technical University of Denmark, Lyngby, Denmark.

Publisher Item Identifier S 0018-926X(97)08806-6.

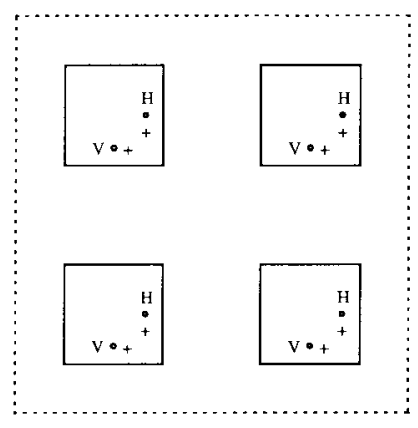

(a)

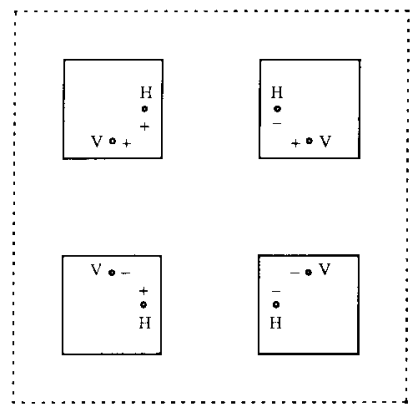

(c)

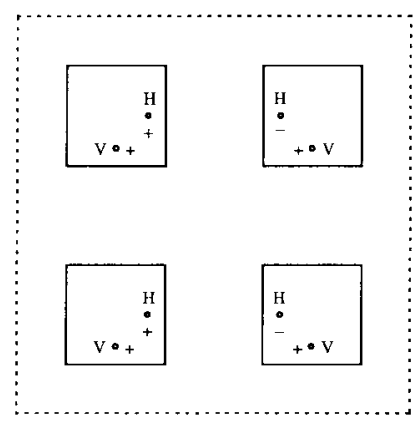

(b)

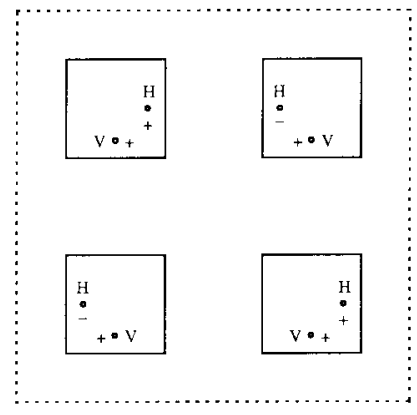

(d)
Fig. 1. $2 \times 2$ element subarray configurations (a)-(d), each consisting of four dual polarization probe-fed square patches with one feeding probe per polarization.

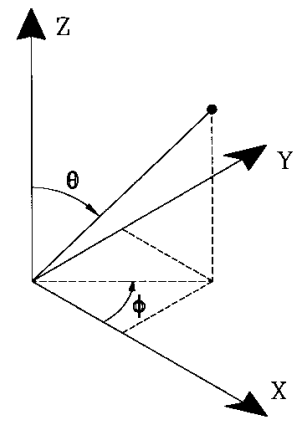

Fig. 2. Coordinate systems.

port should be fed to give all elements in the subarray the same effective excitation (meaning that the co-polar fields for the elements add in phase toward boresight. Ports marked "-" must be fed $180^{\circ}$ out of phase with respect to ports marked "+."

The properties of arrays employing each of the subarray configurations in Fig. 1(a)-(d) are summarized below and a formal treatment is given in the following sections. 

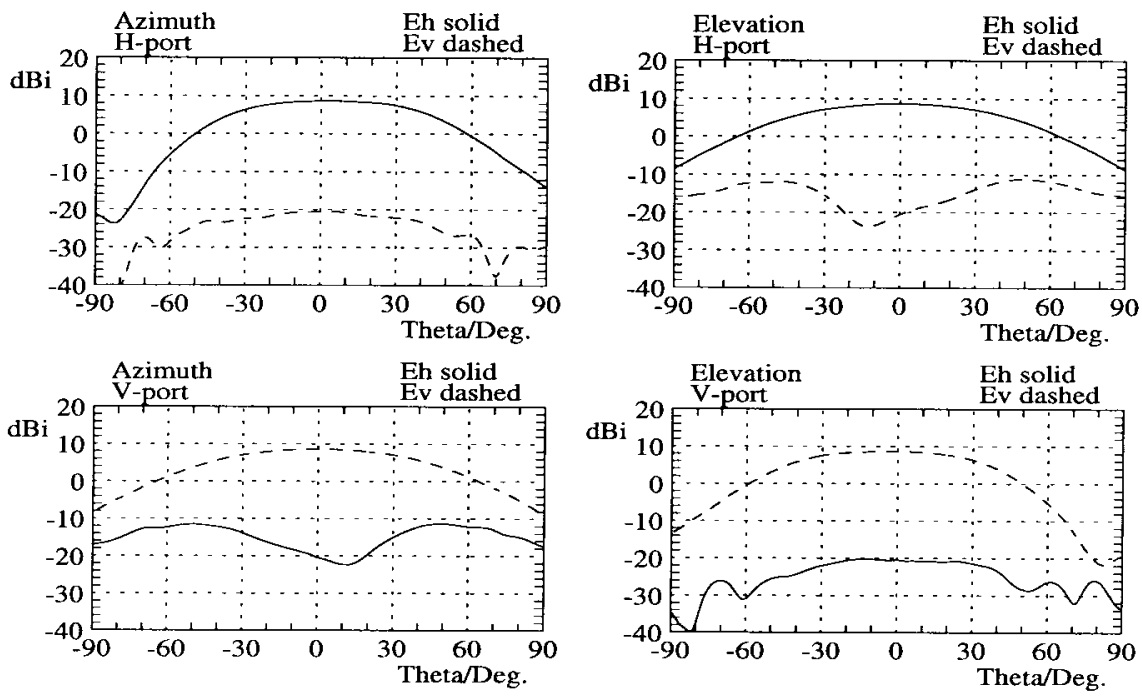

Fig. 3. Measured radiation patterns for dual polarization patch.

In configuration $A$, the individual elements have the same orientation. This is considered the baseline configuration. Using configuration $A$, the array cross-polarization suppression will be exactly as for the single element (ignoring feed network contamination).

In configuration $B$, the two rightmost elements have been mirrored with respect to a vertical plane [2] (where the focus is primarily on the isolation between the antenna ports and not on cross-polarization suppression). It has been applied in several designs (e.g. [3], [4]). Using configuration $B$, the array cross polarization is improved significantly (particularly in the vertical plane), but undesired sidelobes will appear in the horizontal plane.

In configuration $C$, the element mirroring is performed in the horizontal and in the vertical plane. This configuration has also been applied in several designs (e.g. [5], [6]). Using configuration $C$, the array cross-polarization is improved significantly, but undesired sidelobes will appear in the horizontal as well as in the vertical plane.

In configuration $D$, the upper right and the lower left elements are mirrored with respect to a vertical plane [7]. Using configuration $D$, the array cross polarization is improved significantly and the sidelobe problems experienced when using configurations $B$ and $C$ are reduced significantly. Further improvement of the array performance can be obtained by repeated application of the configuration $D$ principle, described later as configuration $E$.

\section{TERMinOLOGY}

In this paper, dual polarization antenna arrays which consist of a number of antenna elements located in a rectangular grid in the $x-y$ plane are considered. The orientation of the spherical coordinate system used to describe the radiation pattern $\bar{E}(\theta, \varphi)$ relative to the rectangular coordinate system used to describe array geometry is shown in Fig. 2. The direction perpendicular to the $x-y$ plane is designated boresight. The $x-z$ plane $(\varphi=0, \theta \in[-\pi ; \pi])$ is the azimuth plane. The $y-z$ plane $(\varphi=\pi / 2, \theta \in[-\pi ; \pi])$ is the elevation plane.

The radiation pattern (electrical far field) from any antenna can be expressed as

$$
\bar{E}(\theta, \varphi)=\left\{\begin{array}{l}
E^{h}(\theta, \varphi) \\
E^{v}(\theta, \varphi)
\end{array}\right\}
$$

where $E^{h}$ and $E^{v}$ are the horizontally and vertically polarized components of the radiation pattern.

Superscripts $H$ and $V$ are used for fields generated by excitation at the $H$ and $V$-ports of an array or array element

$$
\begin{aligned}
& \bar{E}^{H}(\theta, \varphi)=\left\{\begin{array}{l}
E^{H h}(\theta, \varphi) \\
E^{H v}(\theta, \varphi)
\end{array}\right\} \\
& \bar{E}^{V}(\theta, \varphi)=\left\{\begin{array}{l}
E^{V h}(\theta, \varphi) \\
E^{V v}(\theta, \varphi)
\end{array}\right\}
\end{aligned}
$$

The cross-polarization suppression is in this context defined as the ratio between the total and the undesired field components in any direction, i.e., $\left|\bar{E}^{H}(\theta, \varphi)\right| /\left|E^{H v}(\theta, \varphi)\right|$ when the desired polarization is horizontal and $\left|\bar{E}^{V}(\theta, \varphi)\right| /\left|E^{V h}(\theta, \varphi)\right|$ when the desired polarization is vertical. When not explicitly stating a particular direction, the term "cross-polarization suppression" will be used to refer to the level of the crosspolarization suppression in the angular region of interest, e.g., within the main beam of an array.

Any radiation pattern can be separated into its even and odd symmetry components with respect to the elevation plane

$$
\begin{aligned}
\bar{E}(\theta, \varphi) & =\left\{\begin{array}{l}
E^{h}(\theta, \varphi) \equiv E^{h e}(\theta, \varphi)+E^{h o}(\theta, \varphi) \\
E^{v}(\theta, \varphi) \equiv E^{v e}(\theta, \varphi)+E^{v o}(\theta, \varphi)
\end{array}\right\} \\
E^{h e}(\theta, \varphi) & =E^{h e}(\theta, \pi-\varphi), E^{h o}(\theta, \varphi)=-E^{h o}(\theta, \pi-\varphi) \\
E^{v e}(\theta, \varphi) & =E^{v e}(\theta, \pi-\varphi), E^{v o}(\theta, \varphi)=-E^{v o}(\theta, \pi-\varphi)
\end{aligned}
$$

It should be noted that in the elevation plane we have

$$
\bar{E}(\theta, \pi / 2)=\left\{\begin{array}{l}
E^{h}(\theta, \pi / 2)=E^{h e}(\theta, \pi / 2) \\
E^{v}(\theta, \pi / 2)=E^{v e}(\theta, \pi / 2)
\end{array}\right\} .
$$

These even/odd symmetry properties will be used below to calculate radiation properties for different array configurations. 

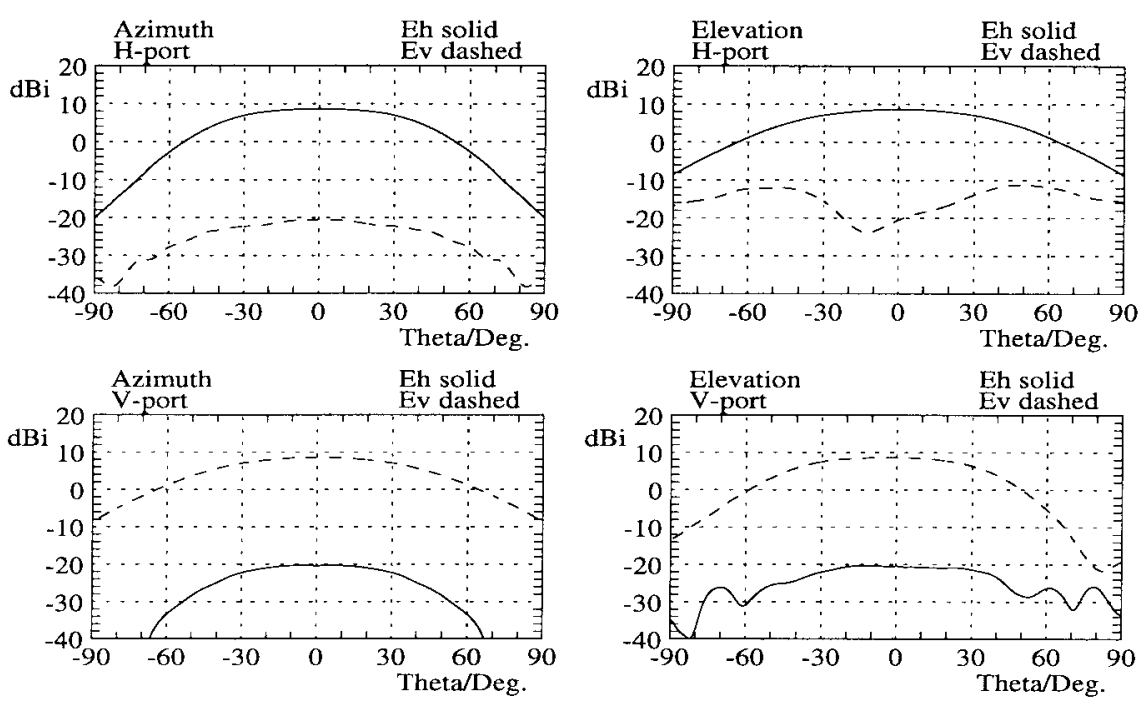

(a)
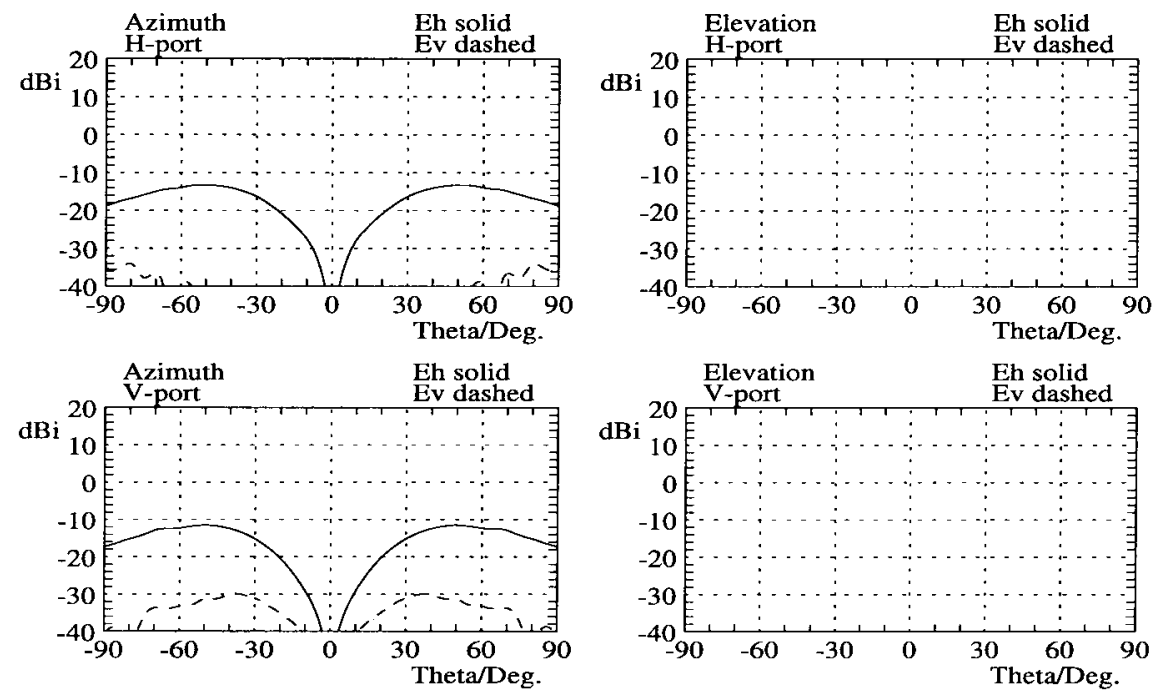

(b)

Fig. 4. (a) Even and (b) odd field components for dual polarization patch.

\section{ARRAY PROPERTIES}

The radiation pattern of an array of antenna elements can be found from

$$
\begin{aligned}
\bar{E}_{\text {Array }}(\theta, \varphi) & =\sum_{i} G_{i}(\theta, \varphi) \bar{E}_{i}(\theta, \varphi) \\
G_{i}(\theta, \varphi) & =A_{i} e^{j P_{i}(\theta, \varphi)} \\
P_{i}(\theta, \varphi) & =k_{0} \bar{r}_{i} \cdot \hat{n} \\
\bar{r}_{i} & =\left(x_{i}, y_{i}, z_{i}\right) \\
\hat{n} & =(\sin \theta \cos \varphi, \sin \theta \sin \varphi, \cos \theta) \\
k_{0} & =2 \pi / \lambda_{0} .
\end{aligned}
$$

$\bar{E}_{i}(\theta, \varphi), \bar{r}_{i}$, and $A_{i}$ are the radiation pattern, location, and excitation of the $i^{\prime}$ th element in the array, respectively. $\lambda_{0}$ is the free-space wavelength. In the following, it is assumed that all elements are located in the $x-y$ plane, i.e., $z_{i}=0$, and that the array center is at $x=y=0$.

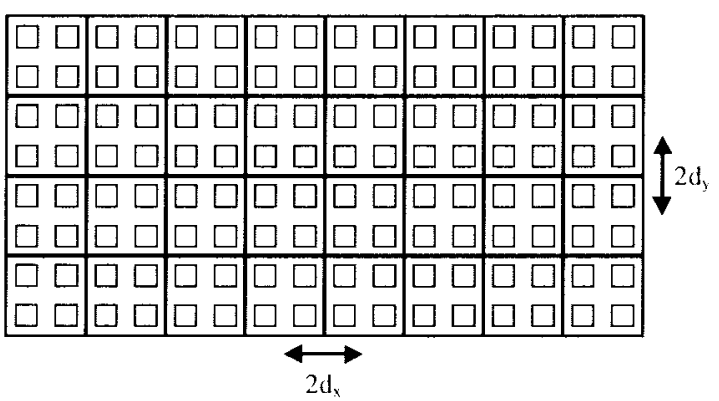

Fig. 5. $16 \times 8$ element array organized as $8 \times 4$ identical $2 \times 2$ element subarrays.

If all elements are identical we have

$$
\begin{aligned}
\bar{E}_{\text {Array }}(\theta, \varphi) & =G(\theta, \varphi) \bar{E}_{\text {Element }}(\theta, \varphi) \\
G(\theta, \varphi) & =\sum_{i} G_{i}(\theta, \varphi) .
\end{aligned}
$$




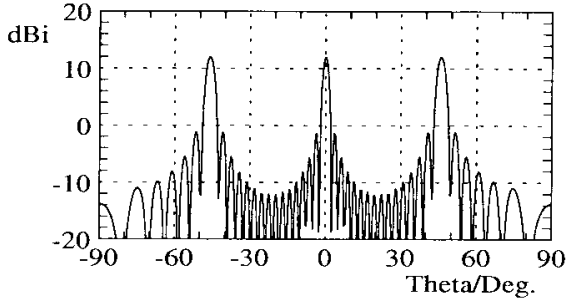

Fig. $6.8 \times 4$ group factor, azimuth and elevation.

Furthermore, if the array is uniformly excited (constant $A_{i}$ ) and the elements are arranged in a regular $M \times N$ grid with spacing $d_{x}$ and $d_{y}$ we find

$$
\begin{aligned}
\bar{E}_{\text {Array }}(\theta, \varphi) & =G_{M \times N}(\theta, \varphi) \bar{E}_{\text {Element }}(\theta, \varphi) \\
G_{M \times N}(\theta, \varphi) & =G_{A z}(\theta, \varphi) G_{E l}(\theta, \varphi) \\
G_{A z}(\theta, \varphi) & =\frac{1}{\sqrt{M}} \sum_{i=0}^{M-1} e^{j k_{0}\left(i-\frac{M-1}{2}\right) d_{x} \cos \varphi \sin \theta} \\
& =\frac{1}{\sqrt{M}} \frac{\sin \left(M k_{0} \frac{d_{x}}{2} \cos \varphi \sin \theta\right)}{\sin \left(k_{0} \frac{d_{x}}{2} \cos \varphi \sin \theta\right)} \\
G_{E l}(\theta, \varphi) & =\frac{1}{\sqrt{N}} \sum_{i=0}^{N-1} e^{j k_{0}\left(i-\frac{N-1}{2}\right) d_{y} \sin \varphi \sin \theta} \\
& =\frac{1}{\sqrt{N}} \frac{\sin \left(N k_{0} \frac{d_{y}}{2} \sin \varphi \sin \theta\right)}{\sin \left(k_{0} \frac{d_{y}}{2} \sin \varphi \sin \theta\right)}
\end{aligned}
$$

The excitations $A_{i}$ have been normalized such that the sum of the squared magnitudes is equal to one (conservation of power).

\section{AnTEnNa ElEMENT}

All calculated array radiation patterns shown in this paper are (unless explicitly stated) based on the radiation pattern of a dual polarization probe-fed stacked microstrip patch, which was used in an $L$-band SAR antenna [8], [9]. The pattern was obtained by measurements in a spherical near-field test facility [10] of a single element on a $0.75 \mathrm{~m} \times 0.75 \mathrm{~m}$ ground plane. The location of the feed probes is as shown in Fig. 1(a). During measurement of the $H$-port pattern the $V$-port was terminated in $50 \Omega$ and vice versa.

The element radiation patterns $\bar{E}_{E}^{H}(\theta, \varphi)$ and $\bar{E}_{E}^{V}(\theta, \varphi)$ are shown in Fig. 3 for the azimuth and elevation planes. The azimuth and elevation patterns including the cross-polar field are shown for both the case where the patch is fed at the $H$ port and for the case where the patch is fed at the $V$-port. Note that the co-polar radiation patterns are asymmetrical in the $E$ plane. This is due to the radiation from the feed probe. The cross-polarization suppression at boresight for the element is $\approx 25 \mathrm{~dB}$, which is not satisfactory for polarimetric SAR applications.

The even and odd field components according to (4) are shown in Fig. 4. The even co-polar field components $E^{\text {Hhe }}$ and $E^{V v e}$ are the dominant ones. The even cross-polar field components $E^{H v e}$ and $E^{V h e}$ are primarily due to excitation of the orthogonal polarization caused by coupling between the $H$ and $V$-ports. The odd $h$-polarization components $E^{H h o}$

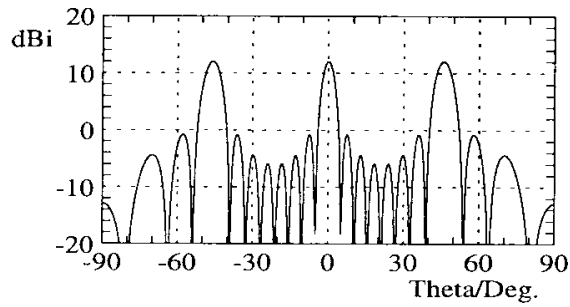

and $E^{V h o}$ are primarily due to radiation from the feed probe. The odd $v$-polarization components $E^{H v o}$ and $E^{V v o}$ are small and, therefore, not significant. Elements with other physical characteristics will have different relative magnitudes of the individual even/odd field components.

\section{ARRAY CONFIGURATIONS}

We now consider the properties of antenna arrays consisting of a number of identical subarrays (groups), each containing $2 \times 2$ radiating elements (see Fig. 5).

When the radiation pattern $\bar{E}_{2 \times 2}(\theta, \varphi)$ of the $2 \times 2$ subarray is known, the array radiation pattern $\bar{E}_{\text {Array }}(\theta, \varphi)$ can be found using (7). As an example a uniformly excited $16 \times 8$ element array ( $8 \times 4$ subarray) is used. In this case, we find

$$
\begin{aligned}
\bar{E}_{\text {Array }}(\theta, \varphi) & =G_{8 \times 4}(\theta, \varphi) \bar{E}_{2 \times 2}(\theta, \varphi) \\
G_{8 \times 4}(\theta, \varphi) & =G_{A z}(\theta, \varphi) G_{E l}(\theta, \varphi) \\
G_{A z}(\theta, \varphi) & =\frac{1}{\sqrt{8}} \sum_{i=0}^{7} e^{j k_{0}(i-3.5) 2 d_{x} \cos \varphi \sin \theta} \\
& =\frac{1}{\sqrt{8}} \frac{\sin \left(8 k_{0} d_{x} \cos \varphi \sin \theta\right)}{\sin \left(k_{0} d_{x} \cos \varphi \sin \theta\right)} \\
G_{E l}(\theta, \varphi) & =\frac{1}{\sqrt{4}} \sum_{i=0}^{3} e^{j k_{0}(i-1.5) 2 d_{y} \sin \varphi \sin \theta} \\
& =\frac{1}{\sqrt{4}} \frac{\sin \left(4 k_{0} d_{y} \sin \varphi \sin \theta\right)}{\sin \left(k_{0} d_{y} \sin \varphi \sin \theta\right)} .
\end{aligned}
$$

In the calculations below $d_{x}=d_{y}=0.7 \lambda_{0}$ is used. Fig. 6 shows $G_{8 \times 4}(\theta, \varphi)$ in the azimuth and elevation planes. Note the grating lobes occurring in the azimuth plane $(\varphi=0)$ for $\theta=\theta_{B}$ where $k_{0} d_{x} \cos \varphi \sin \theta_{B}= \pm \pi$, i.e., $\theta_{B} \cong \pm 45.6^{\circ}$. In the elevation plane similar grating lobes occur at the same value of $\theta$ because the same subarray spacing $\left(2 d_{x}\right.$ and $\left.2 d_{y}\right)$ has been used in the two directions.

\section{A. Configuration A-Identical Elements}

The radiation pattern $\bar{E}_{2 \times 2, A}(\theta, \varphi)$ for the configuration $A$ group [Fig. 1(a)] is found using (6) and is shown in Fig. 7(a)

$$
\begin{aligned}
\bar{E}_{2 \times 2, A}(\theta, \varphi) & =G_{2 \times 2, A}(\theta, \varphi) \bar{E}_{E}(\theta, \varphi) \\
G_{2 \times 2, A}(\theta, \varphi) & =G_{2 \times 2 A z}(\theta, \varphi) G_{2 \times 2 E I}(\theta, \varphi) \\
G_{2 \times 2 A z}(\theta, \varphi) & =\sqrt{2} \cos B \\
G_{2 \times 2 E I}(\theta, \varphi) & =\sqrt{2} \cos C \\
B & =\frac{k_{0} d_{x}}{2} \cos \varphi \sin \theta \\
C & =\frac{k_{0} d_{y}}{2} \sin \varphi \sin \theta .
\end{aligned}
$$



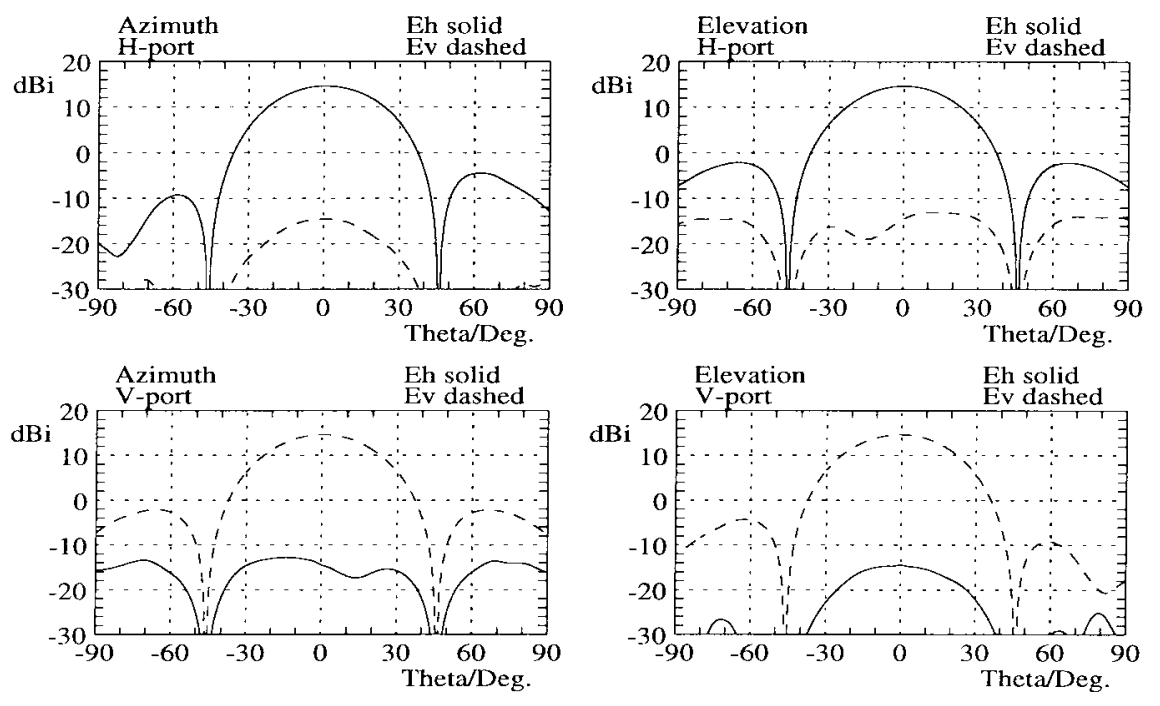

(a)
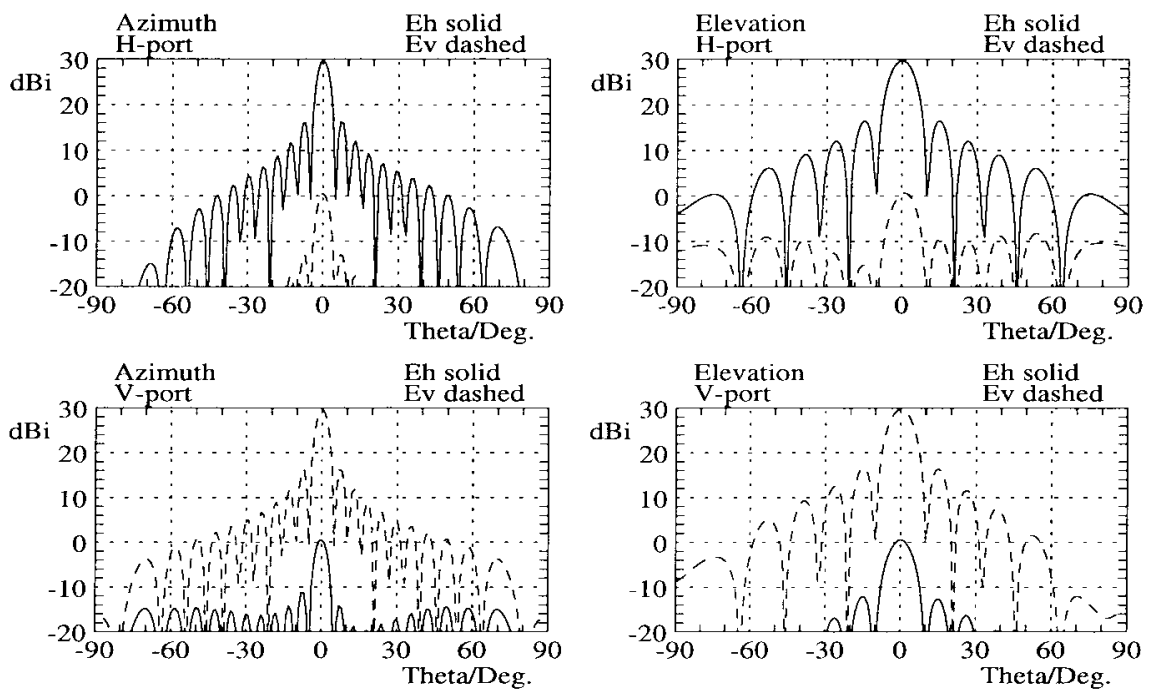

(b)

Fig. 7. Calculated radiation patterns for $2 \times 2$ and $16 \times 8$ element arrays; configuration $A$.

The configuration $A 16 \times 8$ element array radiation pattern $\bar{E}_{\text {Array }, A}(\theta, \varphi)$ is found from $\bar{E}_{2 \times 2, A}(\theta, \varphi)$ using (9) and is shown in Fig. 7(b). The pattern is as expected for uniformly excited and equidistantly spaced arrays of many elements. With respect to cross-polarization properties it is evident that for an array built using identical antenna elements, the ratio between the co- and cross-polar field components is exactly the same as for the single element.

A three-dimensional (3-D) view of the $16 \times 8$ element array radiation pattern is shown in Fig. 11(a). The 3-D figures in Fig. 11 show the magnitude of the radiation pattern in a spherical coordinate system with $0 \mathrm{dBi}$ at the origin, color coded with the cross-polarization suppression. The black lines are equimagnitude contours at $5-\mathrm{dB}$ intervals. The thick black lines are the 10- and 20-dBi contours.

\section{B. Configuration B-The "Single Symmetry" Group}

The field generated by the two upper elements of the configuration $B$ group, Fig. 1(b), is considered first. The left patch is the same as in configuration $A$, whereas the right patch is the mirror image in a vertical plane of the left patch. When vertical polarization is desired, the two $V$-ports must be excited in phase to obtain the same effective excitations of the two elements. When horizontal polarization is desired, the two $H$-ports must be excited $180^{\circ}$ out of phase to obtain the same effective excitations of the two elements.

Since the physical properties of the two patches are each others mirror images, the same must be true for their electrical properties. It is assumed that the field from the left element is given by

$$
\bar{E}_{L}(\theta, \varphi)=\left\{\begin{array}{l}
E_{E}^{h}(\theta, \varphi) \equiv E_{E}^{h e}(\theta, \varphi)+E_{E}^{h o}(\theta, \varphi) \\
E_{E}^{v}(\theta, \varphi) \equiv E_{E}^{v e}(\theta, \varphi)+E_{E}^{v o}(\theta, \varphi)
\end{array}\right\}
$$

If the right element is excited in the same way as the left element, for symmetry reasons we find that

$$
\begin{aligned}
& \bar{E}_{R}(\theta, \varphi) \\
& =\left\{\begin{array}{c}
-E_{E}^{h}(\theta, \pi-\varphi) \equiv-E_{E}^{h e}(\theta, \pi-\varphi)-E_{E}^{h o}(\theta, \pi-\varphi) \\
E_{E}^{v}(\theta, \pi-\varphi) \equiv E_{E}^{v e}(\theta, \pi-\varphi)+E_{E}^{v o}(\theta, \pi-\varphi)
\end{array}\right\} .
\end{aligned}
$$



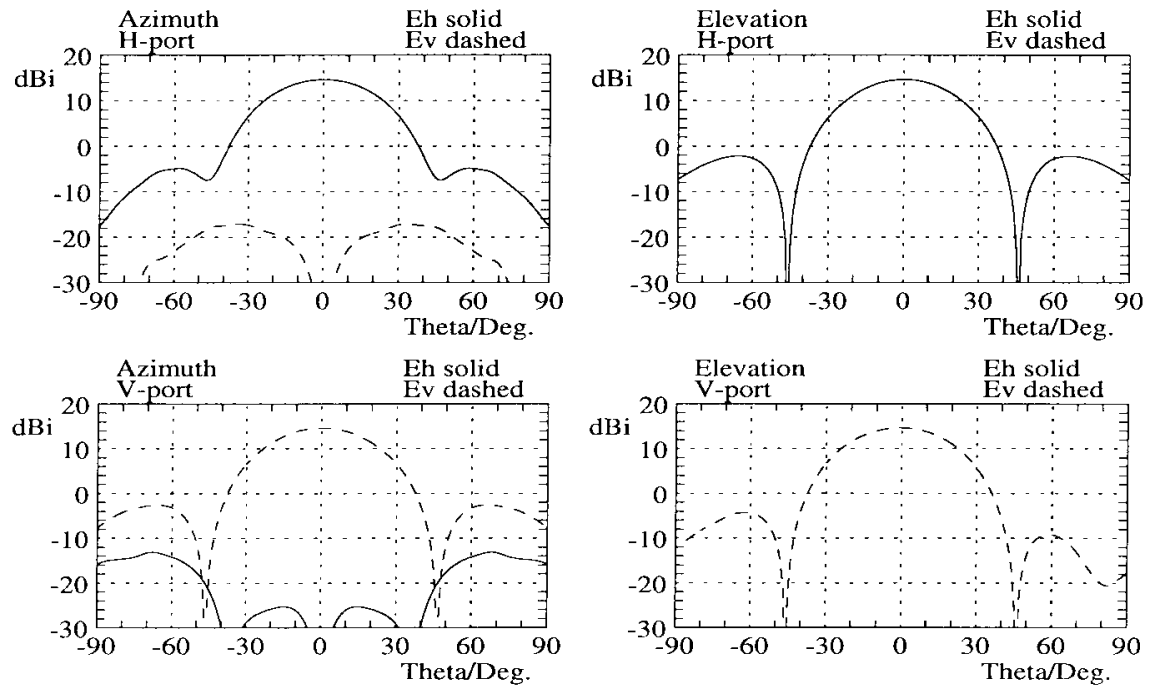

(a)
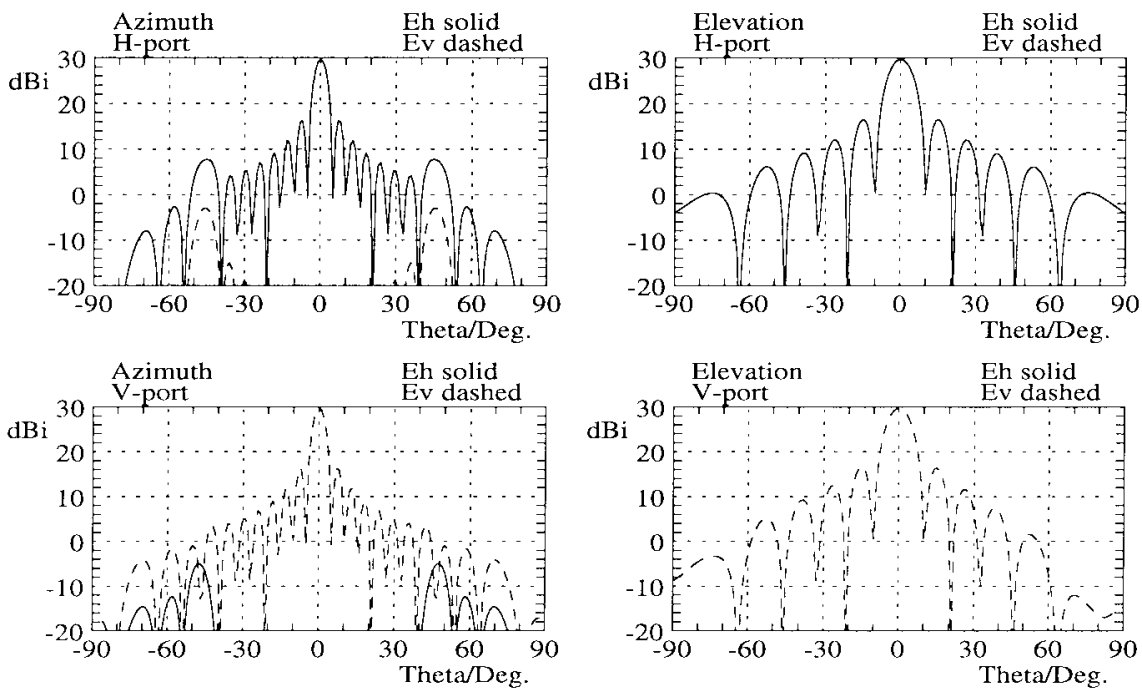

(b)

Fig. 8. Calculated radiation patterns for $2 \times 2$ and $16 \times 8$ element arrays; configuration $B$.

Using the even and odd symmetry properties (4) we find

$$
\bar{E}_{R}(\theta, \varphi)=\left\{\begin{array}{c}
-E_{E}^{h e}(\theta, \varphi)+E_{E}^{h o}(\theta, \varphi) \\
E_{E}^{v e}(\theta, \varphi)-E_{E}^{v o}(\theta, \varphi)
\end{array}\right\} .
$$

The radiation pattern $\bar{E}_{2 \times 1, B U}(\theta, \varphi)$ from the upper two elements is found using (6) and $\left(x_{L}, y_{L}, z_{L}\right)=\left(-d_{x} / 2,0,0\right)$ and $\left(x_{R}, y_{R}, z_{R}\right)=\left(d_{x} / 2,0,0\right)$

$$
\begin{aligned}
\bar{E}_{2 \times 1, B U}(\theta, \varphi) & =G_{L}(\theta, \varphi) \bar{E}_{L}(\theta, \varphi)+G_{R}(\theta, \varphi) \bar{E}_{R}(\theta, \varphi) \\
G_{L}(\theta, \varphi) & =A_{L} e^{-j B} \\
G_{R}(\theta, \varphi) & =A_{R} e^{j B} .
\end{aligned}
$$

$A_{L}$ and $A_{R}$ are the excitations for the left and right element, respectively. When horizontal polarization is desired, the $H$ ports are fed using $A_{L}^{H}=-A_{R}^{H}=\frac{1}{\sqrt{2}}$ and when vertical polarization is desired, the $V$-ports are fed using $A_{L}^{V}=A_{R}^{V}=\frac{1}{\sqrt{2}}$.

Substituting (11) and (13) into (14) for excitation at the $H$-ports we find (15), shown at the bottom of the page. Substituting (11) and (13) into (14) for excitation at the $V$ ports we find (16), shown at the bottom of the next page. The radiation pattern $\bar{E}_{2 \times 2, B}(\theta, \varphi)$ for the $2 \times 2$ element group of Fig. 1(b) is found by multiplying (15) and (16) with the

$$
\begin{aligned}
\bar{E}_{2 \times 1, B U}^{H}(\theta, \varphi) & =\frac{1}{\sqrt{2}}\left(e^{-j B} \bar{E}_{L}^{H}(\theta, \varphi)-e^{j B} \bar{E}_{R}^{H}(\theta, \varphi)\right) \\
& =\frac{1}{\sqrt{2}}\left\{\begin{array}{c}
e^{-j B}\left(E_{E}^{H h e}(\theta, \varphi)+E_{E}^{H h o}(\theta, \varphi)\right)-e^{j B}\left(-E_{E}^{H h e}(\theta, \varphi)+E_{E}^{H h o}(\theta, \varphi)\right) \\
e^{-j B}\left(E_{E}^{H v e}(\theta, \varphi)+E_{E}^{H v o}(\theta, \varphi)\right)-e^{j B}\left(E_{E}^{H v e}(\theta, \varphi)-E_{E}^{H v o}(\theta, \varphi)\right)
\end{array}\right\} \\
& =\sqrt{2}\left\{\begin{array}{c}
\cos B E_{E}^{H h e}(\theta, \varphi)-j \sin B E_{E}^{H h o}(\theta, \varphi) \\
-j \sin B E_{E}^{H v e}(\theta, \varphi)+\cos B E_{E}^{H v o}(\theta, \varphi)
\end{array}\right\}
\end{aligned}
$$



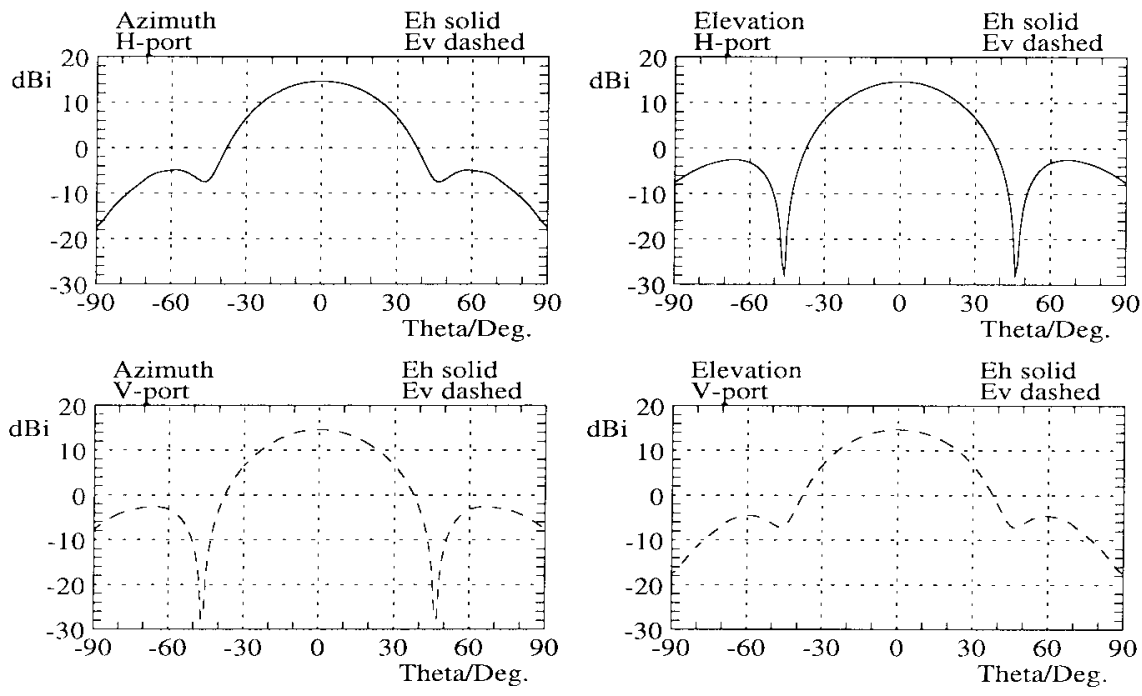

(a)
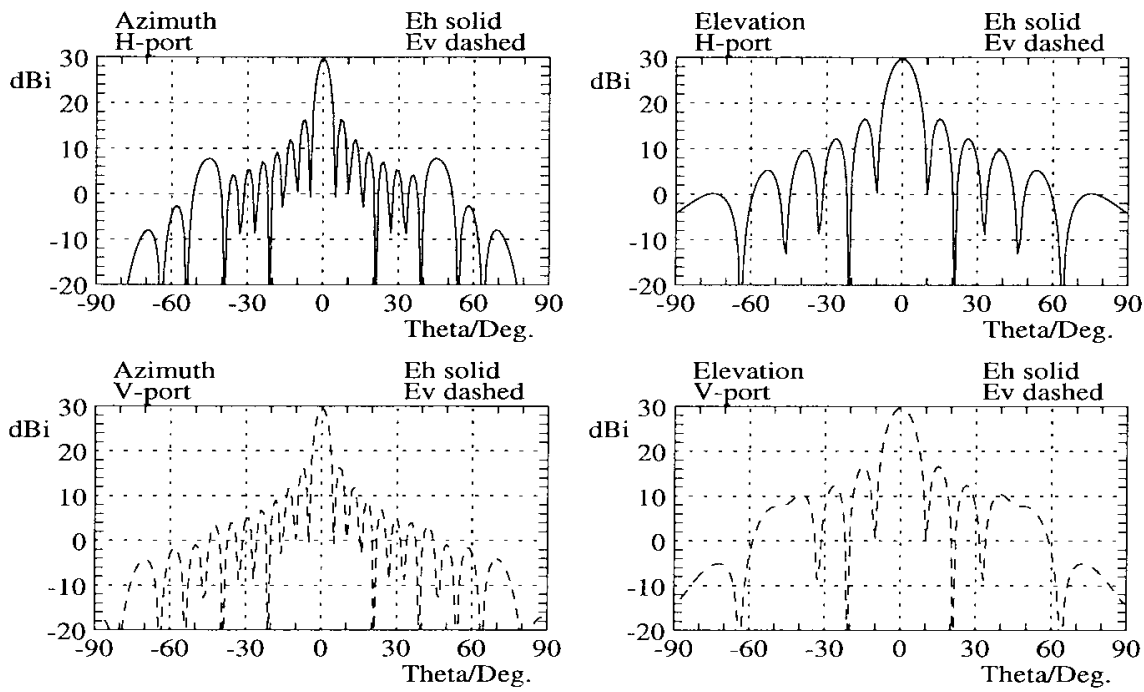

(b)

Fig. 9. Calculated radiation patterns for $2 \times 2$ and $16 \times 8$ element arrays; configuration $C$.

two-element elevation group factor $G_{2 \times 2 E l}$ in (10)

$$
\begin{aligned}
& \bar{E}_{2 \times 2, B}^{H}(\theta, \varphi) \\
& =2 \cos C\left\{\begin{array}{c}
\cos B E_{E}^{H^{v e}}(\theta, \varphi)-j \sin B E_{E}^{H h o}(\theta, \varphi) \\
-j \sin B E_{E}^{H h e}(\theta, \varphi)+\cos B E_{E}^{H v o}(\theta, \varphi)
\end{array}\right\} \\
& \bar{E}_{2 \times 2, B}^{V}(\theta, \varphi) \\
& =2 \cos C\left\{\begin{array}{c}
-j \sin B E_{E}^{V^{h e}}(\theta, \varphi)+\cos B E_{E}^{V^{h o}}(\theta, \varphi) \\
\cos B E_{E}^{V^{2 \epsilon}}(\theta, \varphi)-j \sin B E_{E}^{V^{\circ o}}(\theta, \varphi)
\end{array}\right\} .
\end{aligned}
$$

Azimuth and elevation patterns are shown in Fig. 8(a).
In the elevation plane we have $(\cos B=1, \sin B=0)$

$$
\begin{aligned}
\bar{E}_{2 \times 2, B}^{H}\left(\theta, \frac{\pi}{2}\right) & =2\left\{\begin{array}{c}
\left.\cos C E_{E}^{H h e}\left(\theta, \frac{\pi}{2}\right)\right\} \\
\cos C E_{E}^{H} v o\left(\theta, \frac{\pi}{2}\right)
\end{array}\right\} \\
& =2\left\{\begin{array}{c}
\cos C E_{E}^{H h e}\left(\theta, \frac{\pi}{2}\right) \\
0
\end{array}\right\} \\
\bar{E}_{2 \times 2, B}^{V}\left(\theta, \frac{\pi}{2}\right) & =2\left\{\begin{array}{c}
\cos C E_{E}^{V h o}\left(\theta, \frac{\pi}{2}\right) \\
\cos C E_{E}^{V v e}\left(\theta, \frac{\pi}{2}\right)
\end{array}\right\} \\
& =2\left\{\begin{array}{c}
0 \\
\cos C E_{E}^{V v e}\left(\theta, \frac{\pi}{2}\right)
\end{array}\right\} .
\end{aligned}
$$

$$
\begin{aligned}
\bar{E}_{2 \times 1, B U}^{V}(\theta, \varphi) & =\frac{1}{\sqrt{2}}\left(e^{-j B} \bar{E}_{L}^{V}(\theta, \varphi)+e^{j B} \bar{E}_{R}^{V}(\theta, \varphi)\right) \\
& =\frac{1}{\sqrt{2}}\left\{\begin{array}{l}
e^{-j B}\left(E_{E}^{V h e}(\theta, \varphi)+E_{E}^{V h o}(\theta, \varphi)\right)+e^{j B}\left(-E_{E}^{V h e}(\theta, \varphi)+E_{E}^{V h o}(\theta, \varphi)\right) \\
e^{-j B}\left(E_{E}^{V v e}(\theta, \varphi)+E_{E}^{V o}(\theta, \varphi)\right)+e^{j B}\left(E_{E}^{V v e}(\theta, \varphi)-E_{E}^{V v o}(\theta, \varphi)\right)
\end{array}\right\} \\
& =\sqrt{2}\left\{\begin{array}{l}
-j \sin B E_{E}^{V h e}(\theta, \varphi)+\cos B E_{E}^{V h o}(\theta, \varphi) \\
\cos B E_{E}^{V v e}(\theta, \varphi)-j \sin B E_{E}^{V v o}(\theta, \varphi)
\end{array}\right\}
\end{aligned}
$$



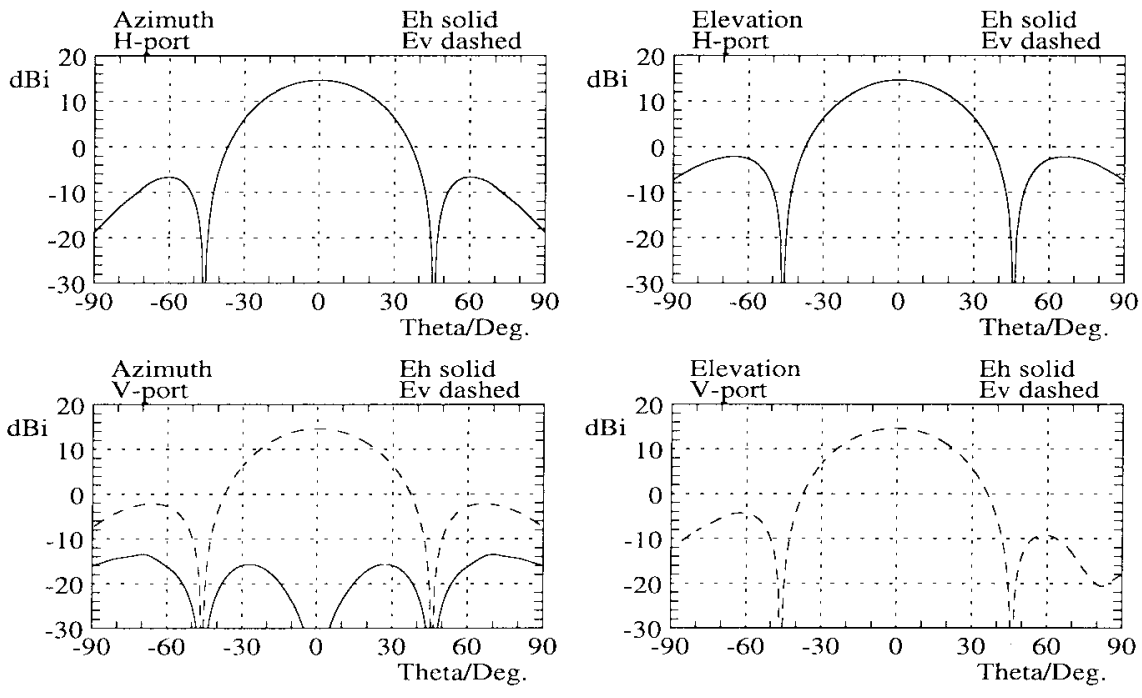

(a)
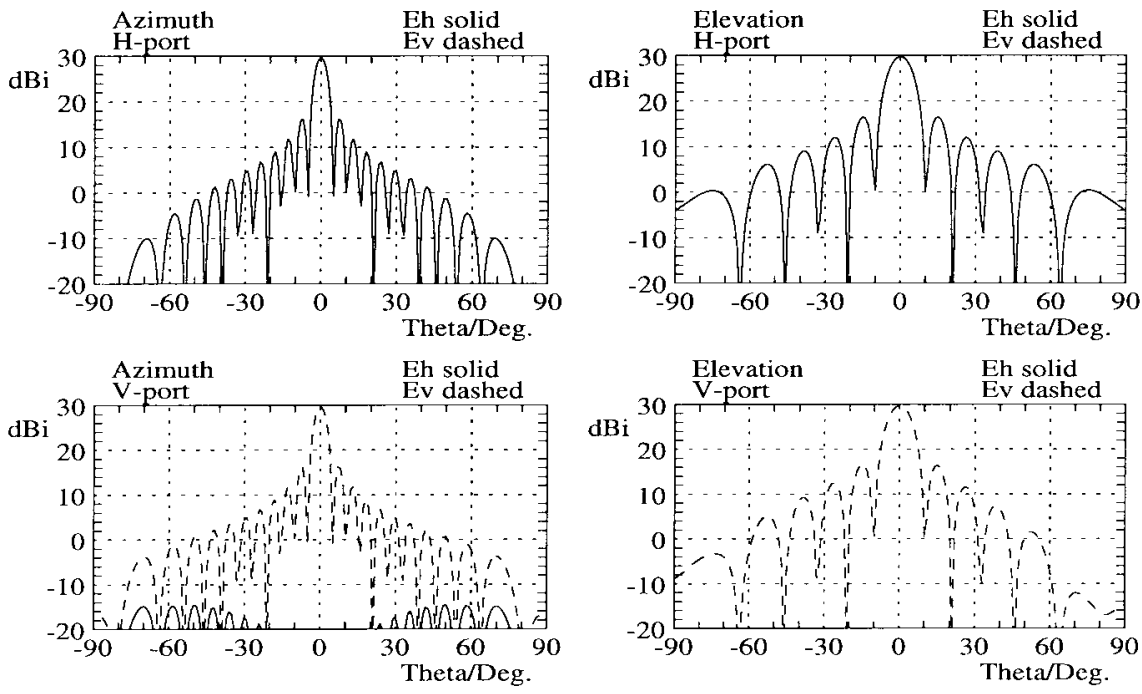

(b)

Fig. 10. Calculated radiation patterns for $2 \times 2$ and $16 \times 8$ element arrays; configuration $D$.

From (18) it is seen that the field from the two-mirrored element group in the elevation plane only contains the desired co-polar field component. In the $H$-polarization case, $E_{E}^{H v e}$ (which is the primary contributor to the cross-polar field) from the two elements cancel each other and similarly with $E_{E}^{V h e}$ in the $V$-polarization case. The cancellation is (in theory) exact in the elevation plane, but also off the elevation plane the undesired field is suppressed. In the azimuth plane, the crosspolarization suppression is also significantly improved, but the cross-polar fields do not vanish.

Comparing Fig. 8(a) with Fig. 7(a) confirms that the crosspolarization suppression has been significantly improved. Thus, by pairing the elements two by two as described above, an antenna array can be designed that has better cross-polarization suppression than the individual antenna element.

The configuration $B 16 \times 8$ element array radiation pattern $\bar{E}_{\text {Array }, B}(\theta, \varphi)$ is found from $\bar{E}_{2 \times 2, B}(\theta, \varphi)$ using (9) and is shown in Fig. 8(b). The co-polar patterns are very similar to the ones for configuration $A$ shown in Fig. 7(b), except that in the azimuth plane a pair of sidelobes has appeared. This occurs at the angle(s) $\theta_{B}$ where the group factor $G_{8 \times 4}(\theta, \varphi)$ has its grating lobes. The reason for the sidelobes appearing when using configuration $B$ is that while the azimuth radiation pattern of the configuration $A 2 \times 2$ element group does have a true null in the azimuth plane for $\theta=\theta_{B}$ due to the $\cos B$ factor in (10), the configuration $B 2 \times 2$ element group does not. These sidelobes will in the following be referred to as "the $2 x$ lobes" because they are due to array properties which have a spatial resolution of two times the element spacing in a particular direction. The $2 x$ lobes are most pronounced in $E^{H}$. The magnitude and polarization of the $2 x$ lobes at $\theta=\theta_{B}$ depend on the particular element used. In the azimuth plane for $\theta=\theta_{B}(\Rightarrow \cos B=0, \sin B=1)$ we have

$$
\begin{aligned}
& \bar{E}_{2 \times 2, B}^{H}\left(\theta_{B}, 0\right)=2\left\{\begin{array}{l}
-j E_{E}^{H h o}\left(\theta_{B}, 0\right) \\
-j E_{E}^{H} v e\left(\theta_{B}, 0\right)
\end{array}\right\} \\
& \bar{E}_{2 \times 2, B}^{V}\left(\theta_{B}, 0\right)=2\left\{\begin{array}{l}
-j E_{E}^{V h e}\left(\theta_{B}, 0\right) \\
-j E_{E}^{V} v o\left(\theta_{B}, 0\right)
\end{array}\right\} .
\end{aligned}
$$



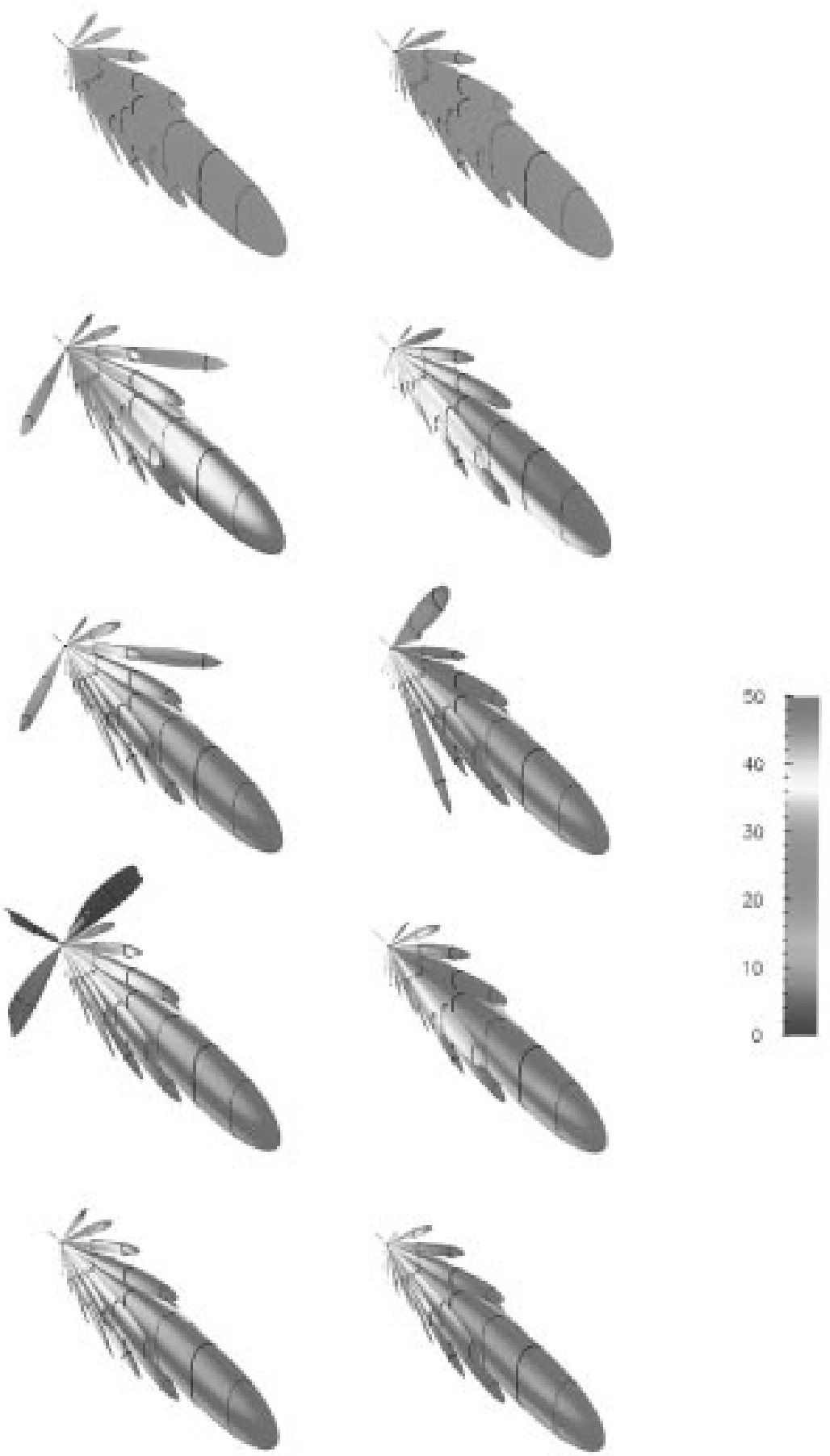

Fig. 11. Calculated 3-D radiation patterns for $16 \times 8$ element arrays; configuration $A-E$. The left and right patterns correspond to feeding at the $H$ and $V$-ports, respectively.

From (19) the sources generating the $2 x$ lobes can be identified.

A 3-D view of the $16 \times 8$ element array radiation patterns is shown in Fig. 11(b). Compared to Fig. 11(a) the crosspolarization suppression is improved significantly over the entire main lobe. The $2 x$ lobes in $E^{H}$ are clearly seen.

The measured radiation pattern of a dual polarization $C$ band SAR antenna $(32 \times 7$ microstrip patch elements) built using configuration $B$ is shown in Fig. 12(a) [4]. The array consists of four $8 \times 7$ element panels which are fed using a $(0.7,1.0,1.0,0.7)$ amplitude taper. In azimuth the element excitations are uniform within each panel. In elevation the excitations are tapered to obtain an approximate modified cosecant squared pattern. It is seen, that the antenna has the expected azimuth $2 x$ lobes (the location is determined by $d_{x}=0.74 \lambda_{0}$ in the $C$-band array). The cross-polar field does not vanish in the elevation plane, but the cross-polarization suppression has been improved from $\approx 25 \mathrm{~dB}$ for the $C$-band patch element to $\approx 30 \mathrm{~dB}$ for the $32 \times 7$ element array. The cross-polar field in the main beam is believed to be caused by a $-30 \mathrm{~dB}$ coupling between the $H$ - and $V$-port feed networks. 

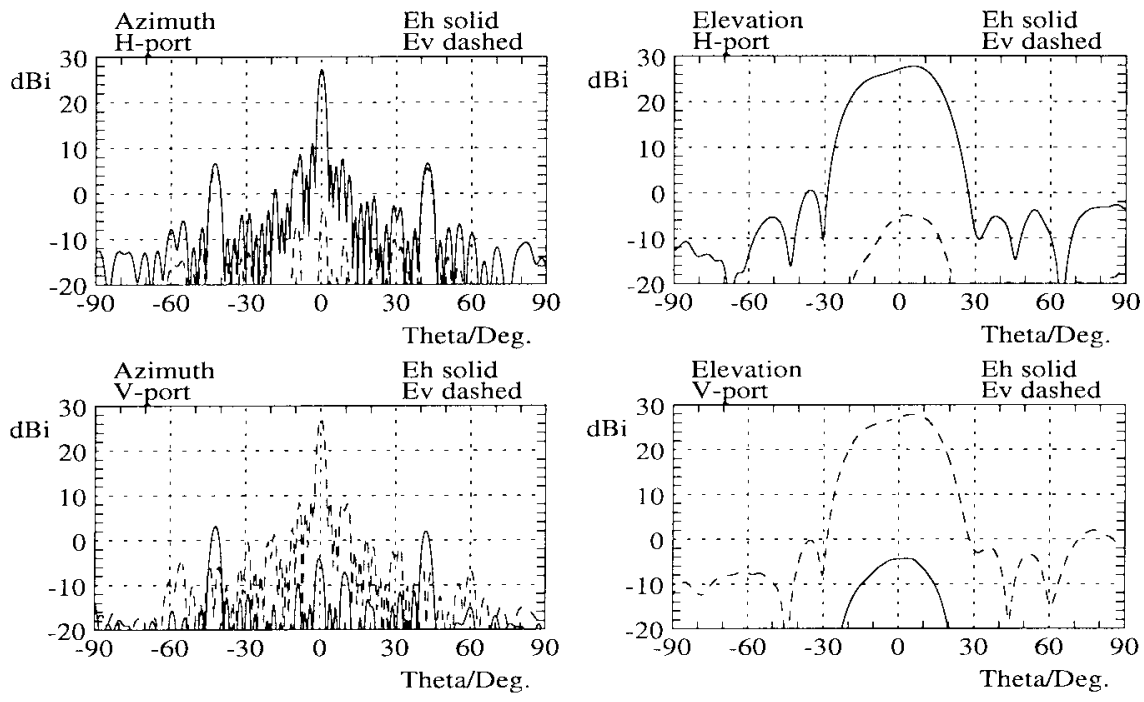

(a)
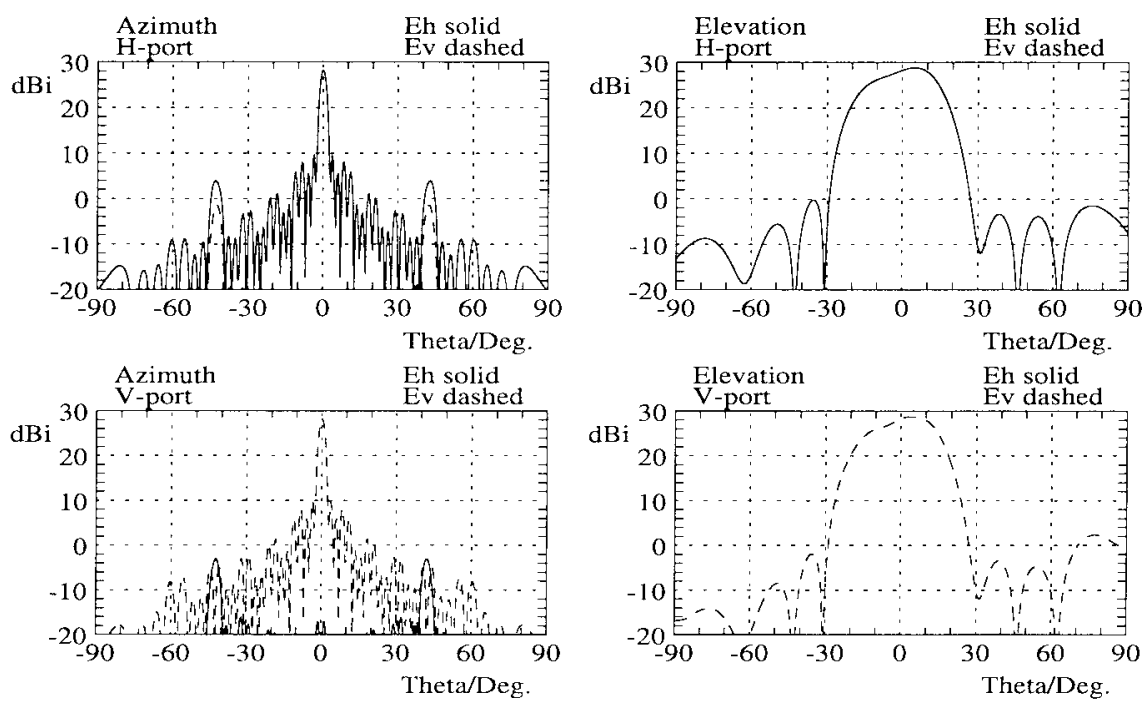

(b)

Fig. 12. (a) Measured and (b) calculated radiation patterns for $32 \times 7$ element $C$-band antenna using configuration $B$.

Fig. 12(b) shows the calculated radiation patterns for the 32 $\times 7$ element $C$-band antenna. The element radiation pattern used in the calculations was obtained by measurements on a single $C$-band patch. Comparing Fig. 12(a) and (b) it is seen that the level of the $2 x$ lobes is somewhat higher in the measured pattern. The increased level of the $2 x$ lobes can be reproduced in calculations if it is assumed that there is a -20 dB coupling between the $H$ and $V$-ports of each element. This coupling may be caused by coupling in the microstrip feed network or by mutual coupling effects.

\section{Configuration C-The "Dual Symmetry" Group}

In the configuration $C 2 \times 2$ element group shown in Fig. 1(c) the principle of mirroring pairs of elements in the elevation plane is also applied in the azimuth plane. The radiation pattern $\bar{E}_{2 \times 2, C}(\theta, \varphi)$ can be found in a similar way as shown for the configuration $B$ group (involving azimuth plane symmetry properties). The result is shown in Fig. 9(a). It is seen that the "missing null" in the $E^{H}$ azimuth pattern for the configuration $B$ group now also exists in the $E^{V}$ elevation pattern for the configuration $C$ group. The cross polarization suppression is very good; it can be shown that the cross-polar fields vanish in both the azimuth and elevation planes. Because the cross-polarization vanishes in the azimuth and elevation planes, the $2 x$ lobes are co-polar.

The configuration $C 16 \times 8$ element array radiation pattern $\bar{E}_{\text {Array }, C}(\theta, \varphi)$ is found from $\bar{E}_{2 \times 2, C}(\theta, \varphi)$ using (9) and is shown in Fig. 9(b). The array pattern now has $2 x$ lobes in both the azimuth plane ( $H$ polarization) and in the elevation plane ( $V$ polarization). A 3 -D view of the $16 \times 8$ element array radiation patterns is shown in Fig. 11(c).

\section{Configuration D-The "Broken Symmetry" Group}

We propose the configuration $D 2 \times 2$ element group shown in Fig. 1(d). The upper element pair is identical to that of the configuration $B$ group, whereas the lower element pair has the individual elements swapped. 

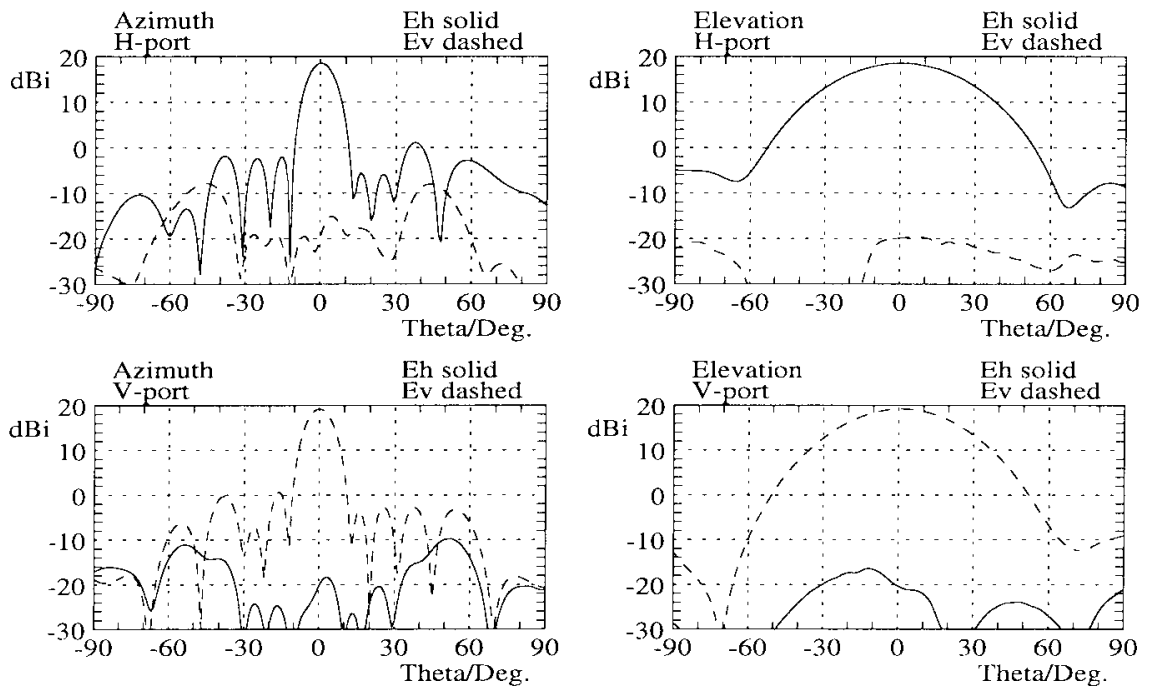

(a)
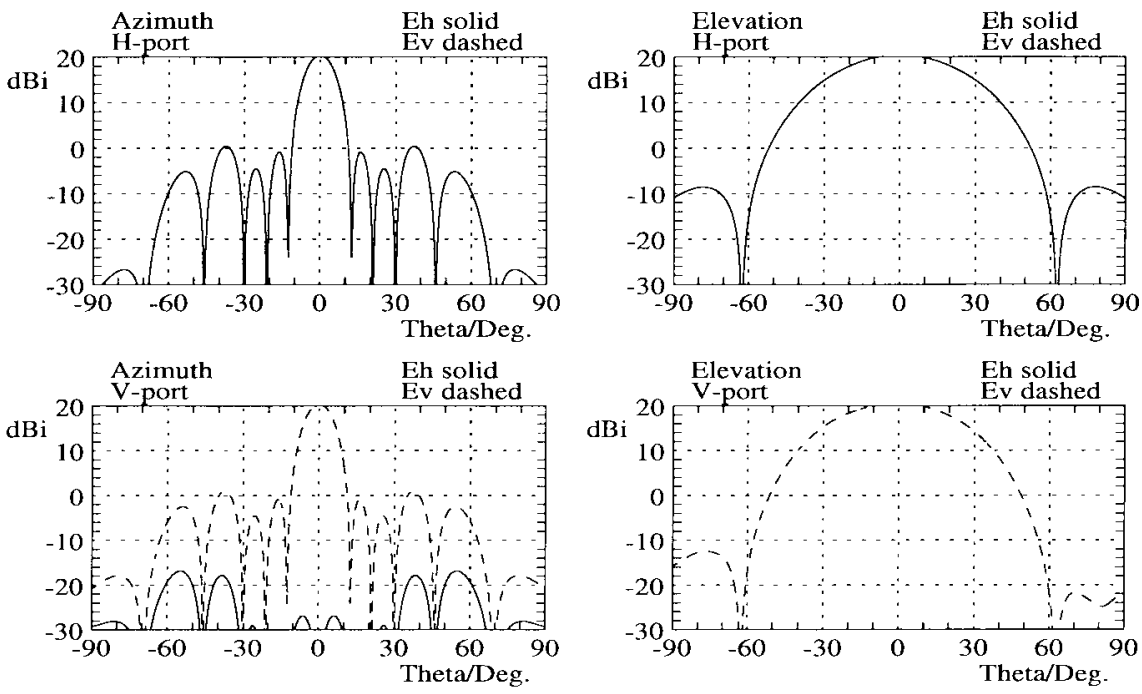

(b)

Fig. 13. (a) Measured and (b) calculated radiation patterns for $8 \times 2$ element $L$-band antenna using configuration $D$.

The radiation pattern for the upper two elements was found in (15) and (16). The field from the lower two-mirroredelement group is found by reversing the sign of $B$

$$
\begin{aligned}
& \bar{E}_{2 \times 1, D L}^{H}(\theta, \varphi) \\
& =\sqrt{2}\left\{\begin{array}{l}
\cos B E_{E}^{H h e}(\theta, \varphi)+j \sin B E_{E}^{H h o}(\theta, \varphi) \\
j \sin B E_{E}^{H v e}(\theta, \varphi)+\cos B E_{E}^{H} v o(\theta, \varphi)
\end{array}\right\} \\
& \bar{E}_{2 \times 1, D L}^{V}(\theta, \varphi) \\
& =\sqrt{2}\left\{\begin{array}{l}
j \sin B E_{E}^{V h e}(\theta, \varphi)+\cos B E_{E}^{V h o}(\theta, \varphi) \\
\cos B E_{E}^{V v e}(\theta, \varphi)+j \sin B E_{E}^{V o o}(\theta, \varphi)
\end{array}\right\} .
\end{aligned}
$$

The radiation pattern $\bar{E}_{2 \times 2, D}(\theta, \varphi)$ for the configuration $D 2$ $\times 2$ element group can now be found from

$$
\begin{aligned}
\bar{E}_{2 \times 2, D}(\theta, \varphi)= & \frac{1}{\sqrt{2}} e^{-j C} \bar{E}_{2 \times 1, D L}(\theta, \varphi) \\
& +\frac{1}{\sqrt{2}} e^{j C} \bar{E}_{2 \times 1, B U}(\theta, \varphi) .
\end{aligned}
$$

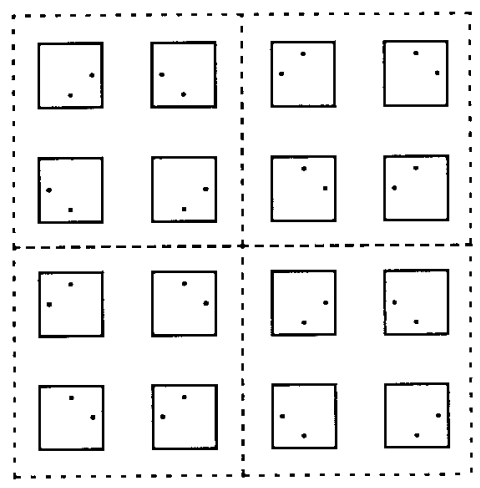

Fig. 14. Configuration $E$ : configuration $D$ applied at $2 \times 2$ element group level.

For excitation at the $H$-ports we find (23), shown at the bottom of the next page. For excitation at the $V$-ports we find (24), shown at the bottom of the next page. $\bar{E}_{2 \times 2, D}(\theta, \varphi)$ is shown in Fig. 10(a). 

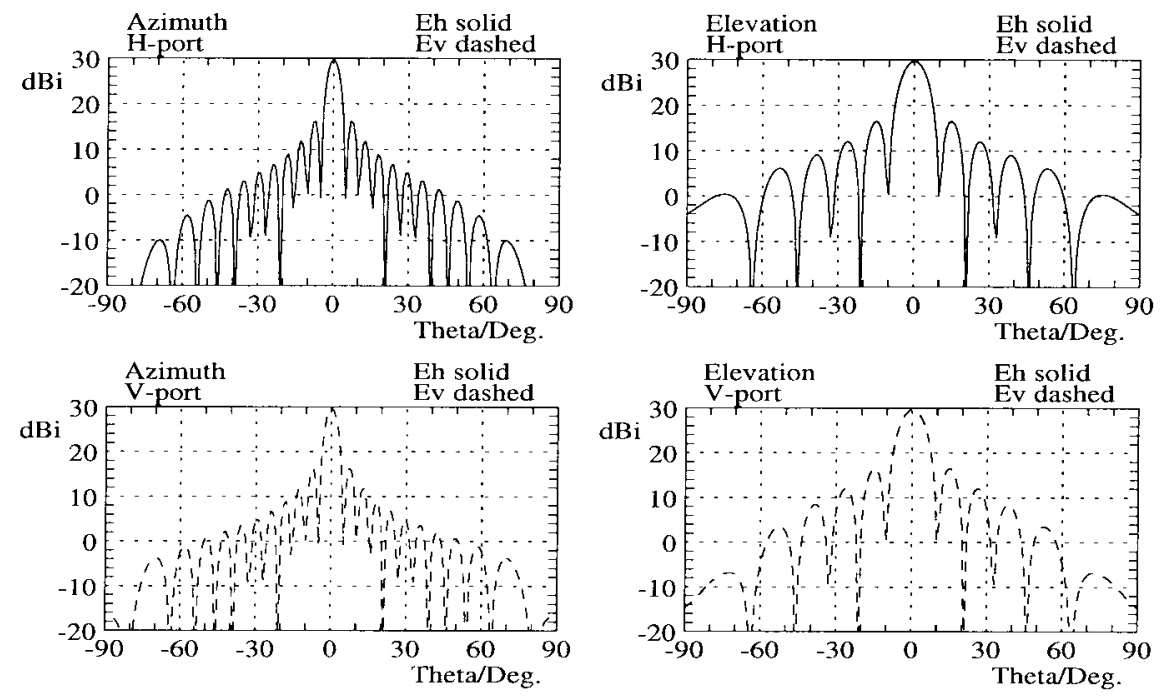

Fig. 15. Calculated radiation patterns for $16 \times 8$ element array; configuration $E$.

In the elevation plane the results are exactly as for configuration $B$, i.e., the cross-polar field is canceled. In the azimuth plane $(\sin C=0, \cos C=1)$ we have

$$
\begin{aligned}
& \bar{E}_{2 \times 2, D}^{H}(\theta, 0)=2\left\{\begin{array}{l}
\cos B E_{E}^{H h e}(\theta, 0) \\
\cos B E_{E}^{H} v o(\theta, 0)
\end{array}\right\} \\
& \bar{E}_{2 \times 2, D}^{V}(\theta, 0)=2\left\{\begin{array}{l}
\cos B E_{E}^{V h o}(\theta, 0) \\
\cos B E_{E}^{V v e}(\theta, 0)
\end{array}\right\} .
\end{aligned}
$$

Comparing (25) with (17) for $\varphi=0$ it is seen that the crosspolar field suppression is improved because the cross-polar field components $E_{E}^{H v e}$ for $V$-port excitation and $E_{E}^{V h e}$ for $H$-port excitation have vanished. Furthermore, when used in an array, the configuration $D$ group will not cause $2 x$ lobes in azimuth, because (by definition) $\cos B=0$ for $\theta=\theta_{B}$.

The configuration $D 16 \times 8$ element array radiation pattern $\bar{E}_{\text {Array }, D}(\theta, \varphi)$ is found from $\bar{E}_{2 \times 2, D}(\theta, \varphi)$ using (9) and is shown in Fig. 10(b); a 3-D view of the $16 \times 8$ element array radiation patterns is shown in Fig. 11(d). The radiation patterns have excellent cross-polarization performance and there are no $2 x$ lobes in azimuth or elevation, however, in the $E^{H}$ pattern four minor grating lobes appear in the diagonal planes (at $\varphi= \pm 45^{\circ}$ due to $d_{x}=d_{y}$ ).

The measured radiation pattern of an $8 \times 2$ element dual polarization $L$-band SAR antenna built using configuration $D$ is shown in Fig. 13(a) [8], [9]. The array consists of four identical panels, each containing a $2 \times 2$ element configuration $D$ group. The elements are the ones used for calculations throughout this paper. The patch feed network, which feeds the four patches in a panel, was designed to give the patches the same effective excitations. The panel feed network was designed to feed the four panels with an amplitude taper with relative magnitudes $(0.6,1.0,1.0,0.6)$ to reduce the azimuth sidelobe level. In elevation an element spacing of $d_{y}=0.56 \lambda_{0}$ was used. The cross-polarization suppression has been improved from $\approx 25 \mathrm{~dB}$ for the single element to $\approx 40 \mathrm{~dB}$ for the $8 \times 2$ element array.

The calculated result obtained by multiplying the four element group pattern $\bar{E}_{2 \times 2, D}(\theta, \varphi)$ (modified for $d_{y}=$ $0.56 \lambda_{0}$ ) by the appropriate group factor is shown in Fig. 13(b). The measured pattern does not have the predicted nulls in elevation for $\theta=\theta_{B}$. The reason is that the ground plane for the real antenna only extends $0.15 \lambda_{0}$ beyond the edges of the patches, causing the radiation patterns for the upper and

$$
\begin{aligned}
\bar{E}_{2 \times 2, D}^{H}(\theta, \varphi) & =\left\{\begin{array}{c}
e^{-j C}\left(\cos B E_{E}^{H h e}(\theta, \varphi)+j \sin B E_{E}^{H h o}(\theta, \varphi)\right)+e^{j C}\left(\cos B E_{E}^{H h e}(\theta, \varphi)-j \sin B E_{E}^{H h o}(\theta, \varphi)\right) \\
e^{-j C}\left(j \sin B E_{E}^{H v e}(\theta, \varphi)+\cos B E_{E}^{H v o}(\theta, \varphi)\right)+e^{j C}\left(-j \sin B E_{E}^{H v e}(\theta, \varphi)+\cos B E_{E}^{H v o}(\theta, \varphi)\right)
\end{array}\right\} \\
& =2\left\{\begin{array}{l}
\cos C \cos B E_{E}^{H h e}(\theta, \varphi)+\sin C \sin B E_{E}^{H h o}(\theta, \varphi) \\
\sin C \sin B E_{E}^{H v e}(\theta, \varphi)+\cos C \cos B E_{E}^{H v o}(\theta, \varphi)
\end{array}\right\}
\end{aligned}
$$

$$
\begin{aligned}
\bar{E}_{2 \times 2, D}^{V}(\theta, \varphi) & =\left\{\begin{array}{c}
e^{-j C}\left(j \sin B E_{E}^{V h e}(\theta, \varphi)+\cos B E_{E}^{V h o}(\theta, \varphi)\right)+e^{j C}\left(-j \sin B E_{E}^{V h e}(\theta, \varphi)+\cos B E_{E}^{V h o}(\theta, \varphi)\right) \\
e^{-j C}\left(\cos B E_{E}^{V v e}(\theta, \varphi)+j \sin B E_{E}^{V v o}(\theta, \varphi)\right)+e^{j C}\left(\cos B E_{E}^{V v e}(\theta, \varphi)-j \sin B E_{E}^{V v o}(\theta, \varphi)\right)
\end{array}\right\} \\
& =2\left\{\begin{array}{l}
\sin C \sin B E_{E}^{V h e}(\theta, \varphi)+\cos C \cos B E_{E}^{V h o}(\theta, \varphi) \\
\cos C \cos B E_{E}^{V v e}(\theta, \varphi)+\sin C \sin B E_{E}^{V v o}(\theta, \varphi)
\end{array}\right\}
\end{aligned}
$$



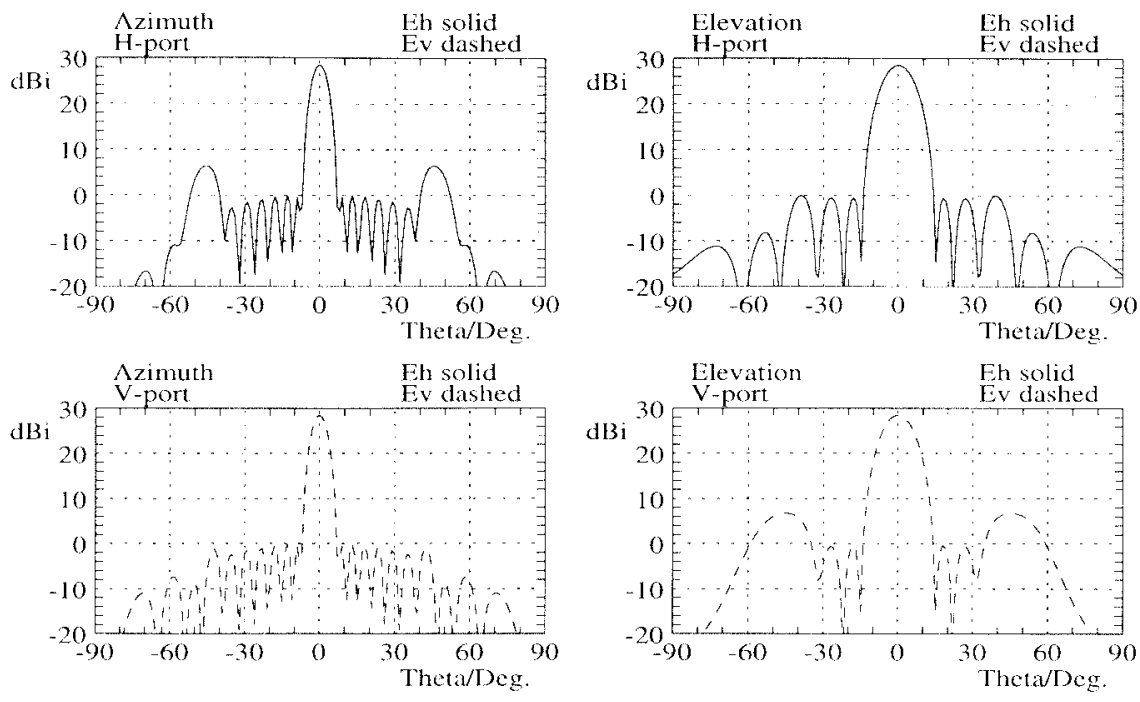

(a)
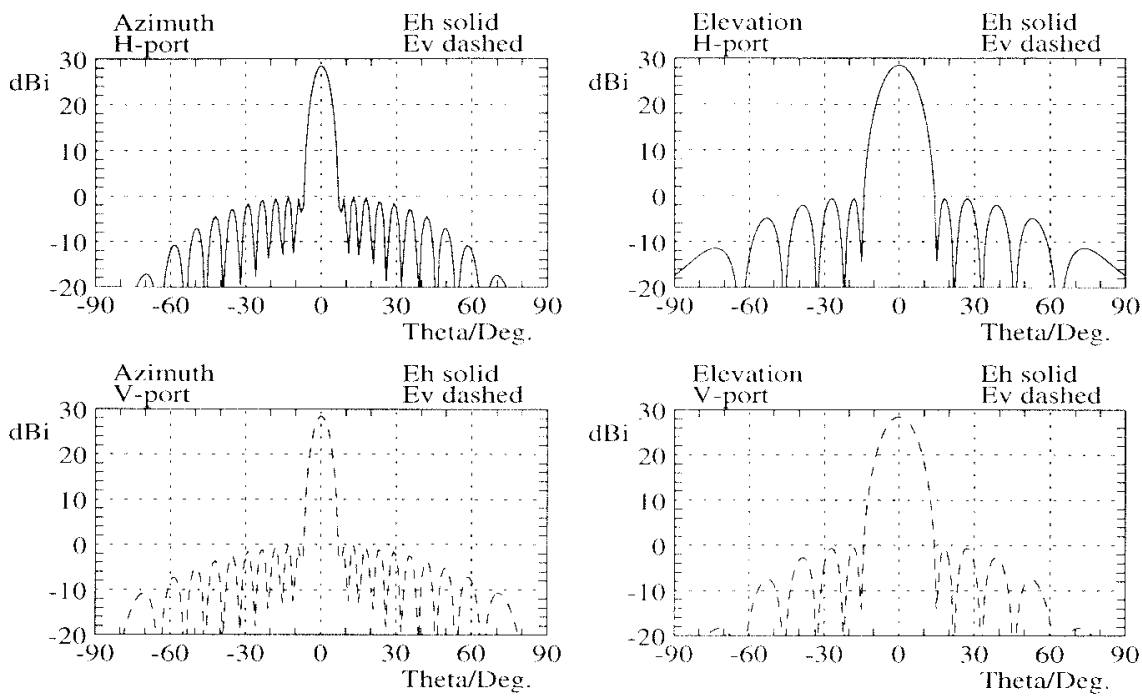

(b)

Fig. 16. Calculated radiation patterns for tapered $16 \times 8$ element array. (a) Configuration $C$. (b) Configuration $E$.

lower patches to be perturbed in opposite directions. This is also believed to be the reason why the cross-polar fields in azimuth are higher than predicted.

\section{E. Application of Configuration D at $2 \times 2$ Element Group Level}

It is possible to make further improvement of the radiation pattern by applying the configuration $D$ principle at the 2 $\times 2$ element group level, e.g., as shown in Fig. 14. This configuration is designated $E$. The plane of symmetry used for mirroring is now the azimuth plane.

The azimuth and elevation radiation patterns of a $16 \times 8$ element configuration $E$ array is shown in Fig. 15 and a 3-D view of the $16 \times 8$ element array radiation patterns is shown in Fig. 11(e). Comparing Fig. 15 with Fig. 10(b) it is seen that for the $E^{H}$ azimuth pattern, the cross-polar field suppression has been improved as the cross-polar fields also vanish in the azimuth plane. The 3-D patterns show that there are no longer any undesired sidelobes.

\section{F. Tapered Excitations}

Previously, uniform excitations have been assumed for all elements in the arrays (except that a panel taper was applied in the implemented $C$ - and $L$-band antennas and that an elevation taper was applied in the implemented $C$-band antenna). In many applications it is desirable to taper the excitations in azimuth as well as in elevation. Fig. 16 shows the radiation patterns for a $16 \times 8$ element array where a Taylor taper has been applied in azimuth and elevation using configurations $C$ and $E$. Note, that in this case, neighboring elements, in general, do not have identical effective excitations.

Comparing Fig. 16(a) and (b) with the corresponding uniformly excited cases (Figs. 9(b) and 15) shows that the qualitative properties with respect to suppression of cross polarization and undesired sidelobes are the same. 


\section{SUMMARY AND FINAL REMARKS}

Various methods for improving the cross-polarization suppression in dual linear polarization antenna arrays and their properties with respect to generation of undesired sidelobes have been discussed. Using simple techniques involving mirroring individual radiating elements or groups of elements, cross-polarization suppression can be improved substantially compared to the properties of the single element and undesired sidelobes can be avoided. Applying a taper to the element excitations does not change these characteristics.

Only dual polarization antenna arrays have been considered. The principles for improving the cross-polarization suppression can also be used in single polarization arrays [7]. Furthermore, it is believed that the principles may find application in reflectarrays.

We have considered only radiation pattern properties, and not issues related to feeding the radiating elements. It is well known that the network feeding the two elements in a mirrored pair can be designed to cancel the coupling between the $H$ and $V$-ports [2]. This effect is very important when designing arrays with good cross-polarization suppression using, for instance, microstrip patches fed by a passive microstrip feed network. If the array consists of active $T / R$ modules (each using a single element as radiator) the issue of isolation between ports is no longer meaningful, but the array radiation pattern can still be improved using the methods described in this paper.

\section{ACKNOWLEDGMENT}

The authors would like to thank Prof. E. L. Christensen and Dr. A. Østergaard of the Department of Electromagnetic Systems, Technical University of Denmark, Lyngby, for their contributions to the preparation of this paper.

\section{REFERENCES}

[1] A. C. Ludwig, "The definition of cross polarization," IEEE Trans. Antennas Propagat., vol. AP-21, pp. 116-119, Jan. 1973.

[2] F. Lalezari and R. E. Munson, "Dual polarized, high efficiency microstrip antenna," U.S. Patent 4464663 , Aug. 1984.

[3] J. Barbero, P. Castillo, Q. García, M. L. Hernanz, and C. Martín-Pascual, "Alternative printed designs for large arrays with dual linear polariza- tion," in Proc. 16th Eur. Space Agency Workshop Dual Polarization Antennas, Noordwijk, The Netherlands, June 1993, pp. 109-116.

[4] J. Granholm, K. Woelders, M. Dich, and E. Lintz Christensen, "Microstrip antenna for polarimetric C-band SAR," IEEE AP-S Int. Symp. URSI Radio Sci. Meet., Seattle, WA, June 1994, pp. 1844-1847.

[5] S. E. Davidson, "Antennas for spacecraft synthetic aperture radars," IEEE Nat. Radar Conf., Los Angeles, CA, Mar. 1986, pp. 31-34.

[6] J. Huang, "Dual-polarized microstrip array with high isolation and low cross-polarization," Microwave Opt. Technol. Lett., vol. 4, no. 3, pp. 99-103, Feb. 1991

[7] J. Granholm and K. Woelders, "Dual polarization antenna array with very low cross polarization and low side lobes," Danish Patent Application 0397/96 no. 661233, filed Apr. 1996.

[8] N. Skou, J. Granholm, K. Woelders, J. Rohde, J. Dall, and E. Lintz Christensen, "A high resolution polarimetric $L$-band SAR-Design and first results," in IGARSS Int. Geosci. Remote Sensing Symp., Firenze, Italy, July 1995, vol. 3, pp. 1779-1782.

[9] J. Granholm and K. Woelders, "Dual polarization microstrip antenna array having very low cross-polarization," Proc. Inst. Elect. Eng. Microwave Antennas Propagat., to be published.

[10] J. E. Hansen and F. Jensen, "Spherical near-field scanning at the Technical University of Denmark," IEEE Trans. Antennas Propagat., vol. 36, pp. 734-739, June 1988.

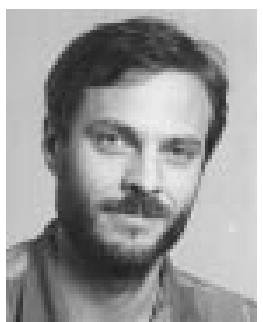

Kim Woelders received the M.Sc. and Ph.D. degrees from the Technical University of Denmark, Lyngby, in 1983 and 1988, respectively.

He is currently a Senior Research Associate at the Department of Electromagnetic Systems, Technical University of Denmark. Since 1986 his work has primarily been concerned with various topics related to the development of the EMISAR system-slotted waveguide array antenna and dual polarization microstrip antenna design, real-time system control computer implementation, system integration, and system operation.

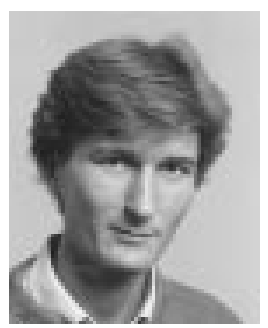

Johan Granholm (M'88) received the M.Sc.E.E degree from the Technical University of Denmark, Lyngby, in 1987.

Since 1987 he has been employed at the Department of Electromagnetic Systems, Technical University of Denmark as a Research Assistant. His main interest has been in development of radio frequency and microwave components and subsystems for the EMISAR system, including dual-polarized microstrip antennas with low cross-polarization. 